Supplementary Information

\title{
Active Patchy Colloids with Shape-Tunable Dynamics
}

Zuochen Wang ${ }^{1}$, Zhisheng Wang ${ }^{1}$, Jiahui Li $^{1}$, Simon Tsz Hang Cheung ${ }^{1}$, Changhao Tian ${ }^{1}$, ShinHyun $\mathrm{Kim}^{2}$ Gi-Ra Yi ${ }^{3}$, Etienne Ducrot ${ }^{4}$ and Yufeng Wang, ${ }^{1, *}$

${ }^{1}$ Department of Chemistry, The University of Hong Kong, Pokfulam Road, Hong Kong SAR, China

${ }^{2}$ Department of Chemical \& Biomolecular Engineering, KAIST, Daejeon 34141, Republic of Korea

${ }^{3}$ School of Chemical Engineering, Sungkyunkwan University, Suwon, 440-746, Republic of Korea

${ }^{4}$ Center for Soft Matter Research, Department of Physics, New York University, New York, New York, 11206, United States

*To whom correspondence should be addressed. E-mail: wanglab@hku.hk

\section{METHODS.}

\section{General methods.}

3-(trimethoxysilyl)propyl methacrylate (TPM) (Sigma-Aldrich), ammonia (Scharlau), sodium dodecyl sulfate (SDS) (J\&K Chemicals), poly(vinylpyrrolidone) (PVP, 29K) (SigmaAldrich), Trition X-100 (TX) (Alfa Aesar), chloroauric acid ( $\mathrm{HAuCl}_{4}$ ) (Dieckmann Chemicals), octadecyltrimethoxysilane (OTMOS) (Dieckmann Chemicals), sodium borohydride $\left(\mathrm{NaBH}_{4}\right)$ (Acros Organics), and formaldehyde (VWR chemicals) were used as received without any further purification. 3-chloro-2-hydroxypropyl methacrylate (CHPMA) (Sigma-Aldrich) was allowed to pass through a column filled with aluminum oxide (basic) to remove the inhibitor. Colloidal particles in the dried state were imaged using a Hitachi S4800 scanning electron microscope (SEM). Bright-field optical images were obtained using a Nikon Eclipse Ti2 inverted microscope 
equipped with a Nikon N7000 camera. Some of the images were digitally post-processed to improve brightness and contrast.

\section{Synthesis of Cl-TPM microspheres.}

Chlorine-functionalized TPM microspheres (Cl-TPM) were synthesized following a reported procedure with modifications ${ }^{1}$. Typically, $3 \mathrm{~mL}$ of TPM monomer was added to $300 \mathrm{~mL}$ of deionized water (DI) containing $300 \mu \mathrm{L}$ of ammonium hydroxide (28\%,w/w). After the mixture was stirred for 4 hours at room temperature, monodisperse micrometer-sized TPM emulsion was produced. Then, $900 \mu \mathrm{L}$ of CHPMA was added and allowed to diffuse into the TPM emulsion. After stirring for another 3 hours, $15 \mathrm{~mL}$ of aqueous SDS solution $(5 \% \mathrm{w} / \mathrm{w})$ was introduced. 30 minutes later, $30 \mathrm{mg}$ of AIBN was added, and the reaction mixture was allowed to stir for another $15 \mathrm{~min}$ before the mixture was put in an oven for heating at $80^{\circ} \mathrm{C}$. Thermal degradation of AIBN initiated the polymerization of the containing monomers. After 4.5 hours, the reaction was quenched by cooling to room temperature, producing Cl-TPM particles $1.2 \mu \mathrm{m}$ in diameter, which were purified by repeated centrifugation and redispersion cycles in DI water. The particle sizes can be adjusted by varying the amount of TPM monomer and ammonia initially added.

\section{Synthesis of $\mathrm{C}_{18}$-TPM microspheres.}

The $\mathrm{C}_{18}$-coated TPM particles were synthesized following the method reported by Yi and coworkers ${ }^{2}$. In a typical case, $p$-TPM (un-functionalized, polymerized TPM) seeds were dispersed in $10 \mathrm{~mL}$ of ethanol, together with $2 \mathrm{~mL}$ of ammonium hydroxide $(28 \%$, w/w), and $2 \mathrm{~mL}$ of diluted OTMOS in chloroform (10 vol\%). The mixture was stirred for 6 hours and ultra-sonicated for three times $\left(20 \mathrm{~s}\right.$ each) to prevent aggregation. The $\mathrm{C}_{18}$-coated TPM suspensions were washed by centrifugation and redispersion cycles in ethanol (three times) and hexanes (three times). Finally, the particles were dispersed in DI water with trace amount of Pluronic F108.

\section{Fabrication of colloidal clusters.}

Clusters consisting of a small number of Cl-TPM microspheres were fabricated using a modified emulsion-evaporation method reported by Manoharan et al. ${ }^{3} \mathrm{Cl}-\mathrm{TPM}$ particles were first transferred into ethanol and then to toluene (containing 5\% of ethanol) by centrifugation and redispersion cycles. The particle suspension diluted to about $1 \%(\mathrm{w} / \mathrm{w})$ was emulsified against $0.1 \%$ 
(w/w) aqueous SDS. After the toluene was removed by first diffusing to hexanes and then by heating, the clusters were obtained and purified by repeated centrifugation and redispersion in DI water. Cl-TPM particles of two different sizes were used to make clusters containing big and small spheres, which were used to synthesize two-patch particles with one big and one small patch.

\section{Synthesis of patchy particles and control of particle shapes by surfactant-aided dewetting.}

$20 \mathrm{~mL}$ of the aqueous suspension of Cl-TPM clusters (roughly $1 \% \mathrm{w} / \mathrm{w}$ ) was charged into a $40 \mathrm{~mL}$ glass vial. Then, $80 \mu \mathrm{L}$ of ammonium hydroxide (28\%) was added, followed by $180 \mu \mathrm{L}$ of TPM monomer. After stirring for $1 \mathrm{~h}, 1.5 \mathrm{~mL}$ of dichloromethane (DCM) was added to merge the multiple TPM droplets nucleated on the surface of cluster. The DCM was then evaporated by gentle heating. Then, a desired amount of Triton X-100 (TX, 10\% aqueous solution) was added to change the particle shape by forcing the TPM droplet to dewet the cluster surface. The extent of dewetting can be controlled by the amount of TX added, and the process can be monitored by optical microscopy immediately after the TX addition. Once the desired geometry was formed, 10 mg AIBN was added. The suspension was stirred for another $15 \mathrm{~min}$ before heating to $80{ }^{\circ} \mathrm{C}$ to initiate the polymerization, which fixed the particle shapes.

\section{Site-specific gold plating.}

The chlorine groups on patchy particle surface were first converted to amine groups, which facilitated the site-specific gold plating on the patches. Typically, $2 \mathrm{~mL}$ of patchy particles suspension $(0.5 \% \mathrm{w} / \mathrm{w})$ containing $0.5 \% \mathrm{~F} 127$ was treated with $1.2 \mathrm{~mL}$ of ethylene diamine and trace amount of potassium iodide (KI). After 4 hours of reaction at $70{ }^{\circ} \mathrm{C}$, amino patchy particles were obtained. The gold plating was done in two steps. In the first step, $120 \mu \mathrm{L}$ of an aqueous chloroauric acid $\left(\mathrm{HAuCl}_{4}\right)$ solution $(4 \mathrm{mg} / \mathrm{mL})$ was added to the patchy particles and the mixture was vortexed for $3 \mathrm{~min}$. After the particle suspension was washed three times with DI water, sodium borohydride $\left(\mathrm{NaBH}_{4}\right)$ was added to reduce the $\mathrm{Au}^{3+}$, forming gold nanoparticles on particle surface. After gently shaking for $5 \mathrm{~min}$, the particle suspension was washed by DI water for another 3 times and dispersed in $0.5 \mathrm{~mL}$ of $0.3 \%$ poly(vinylpyrrolidone) (PVP, $29 \mathrm{~K}$ ) aqueous solution. The gold nanoparticles served as seeds and allowed the growth of the gold layer. For this purpose, we used freshly-made gold hydroxide $\left(\mathrm{Au}(\mathrm{OH})_{3}, 600 \mu \mathrm{L}\right.$ ) (by dissolving $200 \mathrm{mg}$ of $\mathrm{K}_{2} \mathrm{CO}_{3}$ in 20 
$\mathrm{mL}$ of $4 \mathrm{mg} / \mathrm{mL} \mathrm{HAuCl}_{3}$ aqueous solution and stirring overnight) and formaldehyde (HCHO, 67.5 $\mu \mathrm{L}$ ), and the mixture was gently agitated overnight to produce a relatively smooth gold coating.

The coverage of the nanoparticles on the patch surface matters for the particle activity. The origin of activity (e.g., the induced-charge electroosmosis, ICEO) is due to the polarizability difference of the gold particle patch and the dielectric body. If the coverage is low, the amount of gold is not enough to drive the ICEO mechanism (indicated in experiments by very slow propulsion or even no propulsion). The activity of ICEO is increased as the thickness of gold is increased, as discovered by other groups previously ${ }^{4}$. In our experiment, we optimized conditions to produce a relatively high coverage and thick single layer of gold (10-30 nm in diameter), which also ensured fair comparison of activity between different particle shapes.

Compared to the length scale of flow induced dynamics (e.g., induced-charge electroosmotic flow and electrodynamic flow) and interactions (dipole-dipole interaction), which are long-ranged (several micrometers), the roughness due to gold nanoparticles (10-30 nm) has a negligible effect on the mobility and assembly of the system.

\section{Surface Evolver simulation.}

The Surface Evolver package developed by Kenneth Brakke ${ }^{5}$ was used to simulate the structural evolution of patchy particles with different contact angles. From an initial surface defined in a script file, the surface was evolved toward the minimal energy by a gradient descent method, in which each cell on the surface can be progressively refined by a triangular tessellation to achieve the desired level of accuracy. Each iteration step in the Evolver minimized the energy by changing surface in the negative gradient direction of surface energy, until reaching a local minimum.

\section{The propulsion and assembly of patchy particle under AC electric field.}

$20 \mu \mathrm{L}$ of the suspension containing particles of interest was introduced in a cell made by ITO glass (cell height, $50 \mu \mathrm{m}$ ). The ITO glasses were sputter-coated with a thin layer (about $5 \mathrm{~nm}$ ) of iridium to prevent particle sticking to the cell. The electric field by a function generator (RIGOL, DG1022U) was then applied perpendicularly to the ITO plane. Square waves of different voltages and frequencies were used to drive the particle dynamics. For locomotion study, the gold-coated patchy particles were dispersed in $0.1 \mathrm{mM} \mathrm{NaCl}$ aqueous solution. For the assembly study, a 
mixture of 4- $\mu \mathrm{m}$ dielectric spheres (made by $p$-TPM) and gold-coated patchy particles was first prepared and then loaded into the cell. In this case, the medium used was DI water. The dynamics of both locomotion and assembly of the particles were observed on a Nikon Eclipse Ti-2 inverted microscope and video-taped by a Nikon N7000 DSLR camera.

\section{Particle tracking.}

The locomotive trajectories of particles were tracked and processed by Trackpy package with Python.

\section{Numerical simulation.}

COMSOL Multiphysics (Version 5.4a) was used to simulate the induced-charged electroosmotic flow and the local electric field distribution around the gold patchy particles under $\mathrm{AC}$ electric field. In our model, the gap between the top and bottom electrode is set to be $50 \mu \mathrm{m}$, and the micrometer-sized patchy particles are placed $200 \mathrm{~nm}$ above the bottom electrode (by estimating the Debye screen length). We consider a 2D cross-section (a side view) of the model, and include four subdomains, which are $0.1 \mathrm{mM} \mathrm{NaCl}$ aqueous solution, dielectric particle body, the gold-coated patch, and an insulation layer between the dielectric and metallic part of the particle. The electroosmosis velocity is set as the boundary condition of particles. A user-defined mesh is adopted. 
SUPPLEMENTARY FIGURES.

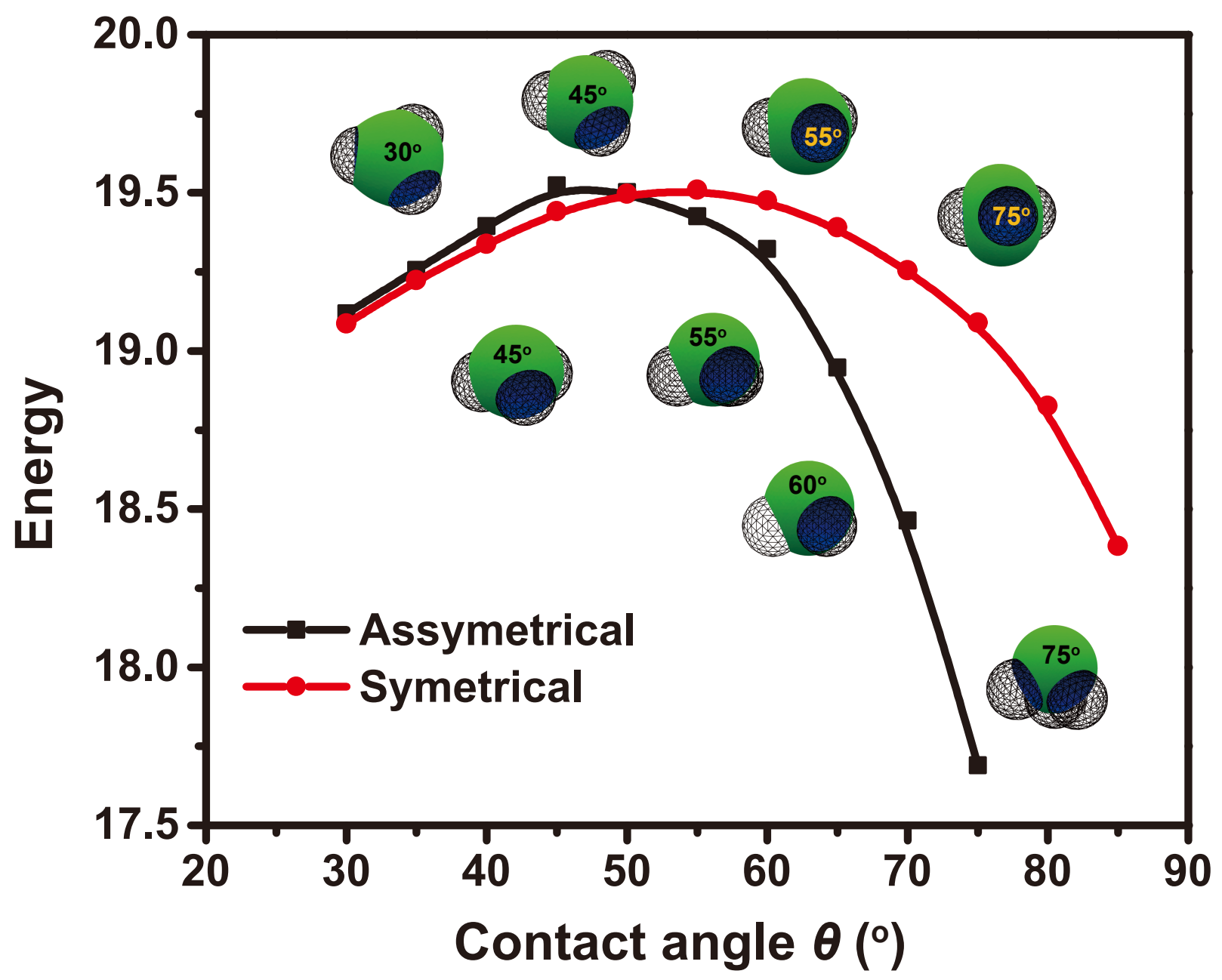

Figure S1. Surface Evolver simulation of the morphologies of three-patch particles. Comparison is made between symmetrical and asymmetrical patch configurations as contact angle is increased. The unit of energy is the surface energy of a seed sphere. 


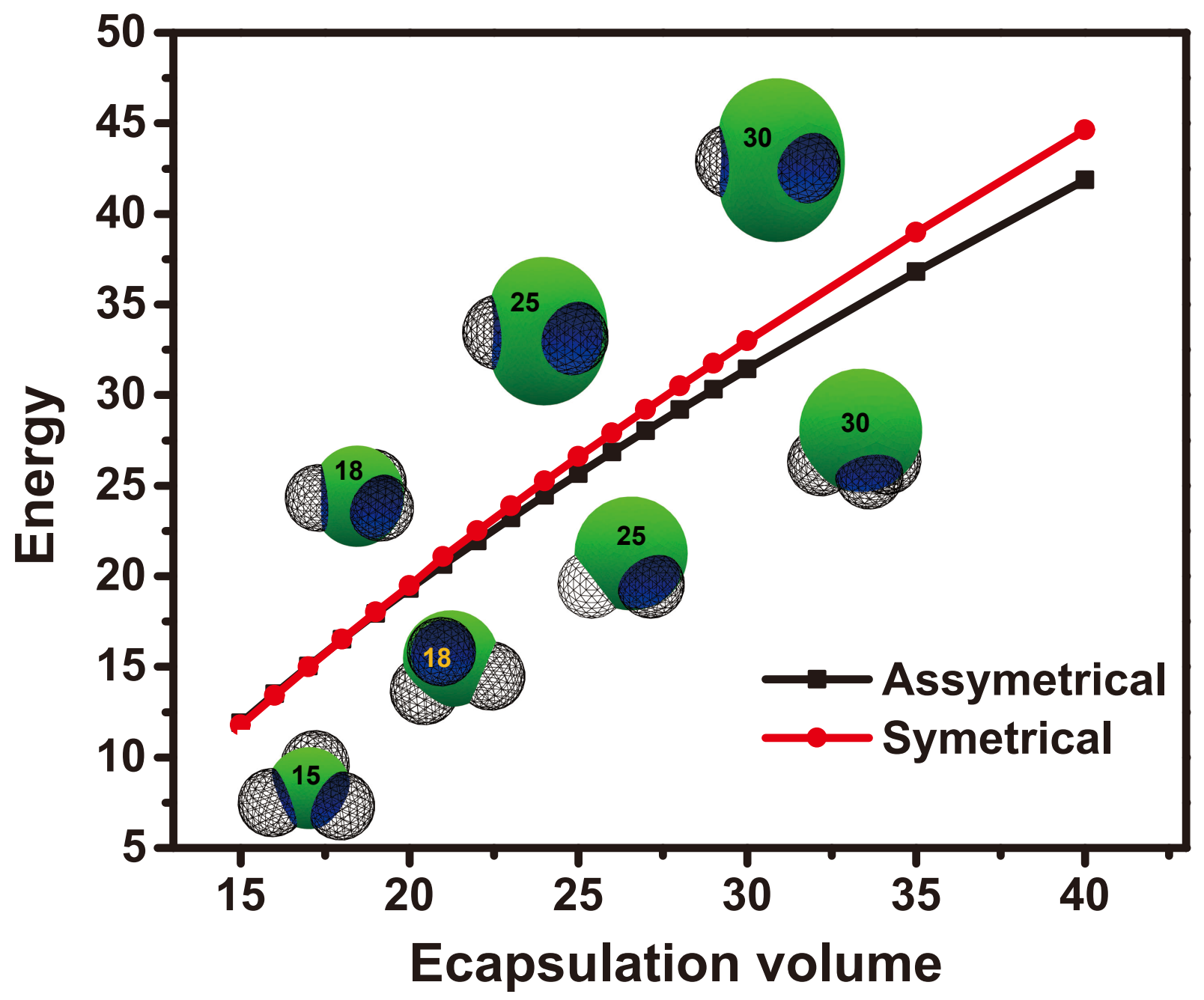

Figure S2. Surface Evolver simulation of the morphologies of three-patch particles. Comparison is made between symmetrical and asymmetrical patch configurations as encapsulation volume is increased (from 15 to 40). The unit of volume is the volume of a seed sphere. 

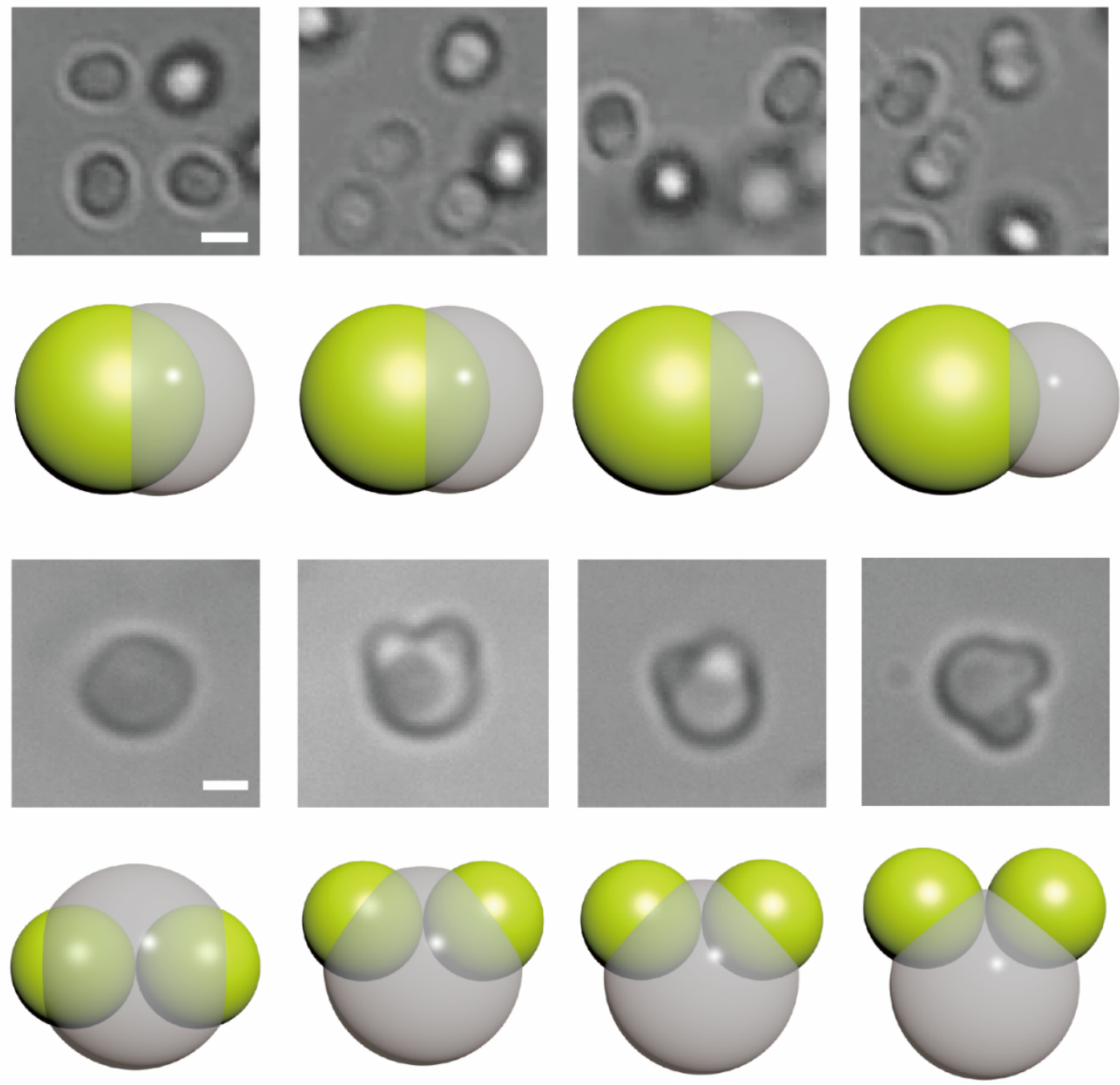

\section{Conc. of TX-100}

Figure S3. In situ optical microscope observation of the morphology evolution as the amount of TX is increased. Top, monopatch particles. Bottom, two-patch particles. Scale bar: $1 \mu \mathrm{m}$. 


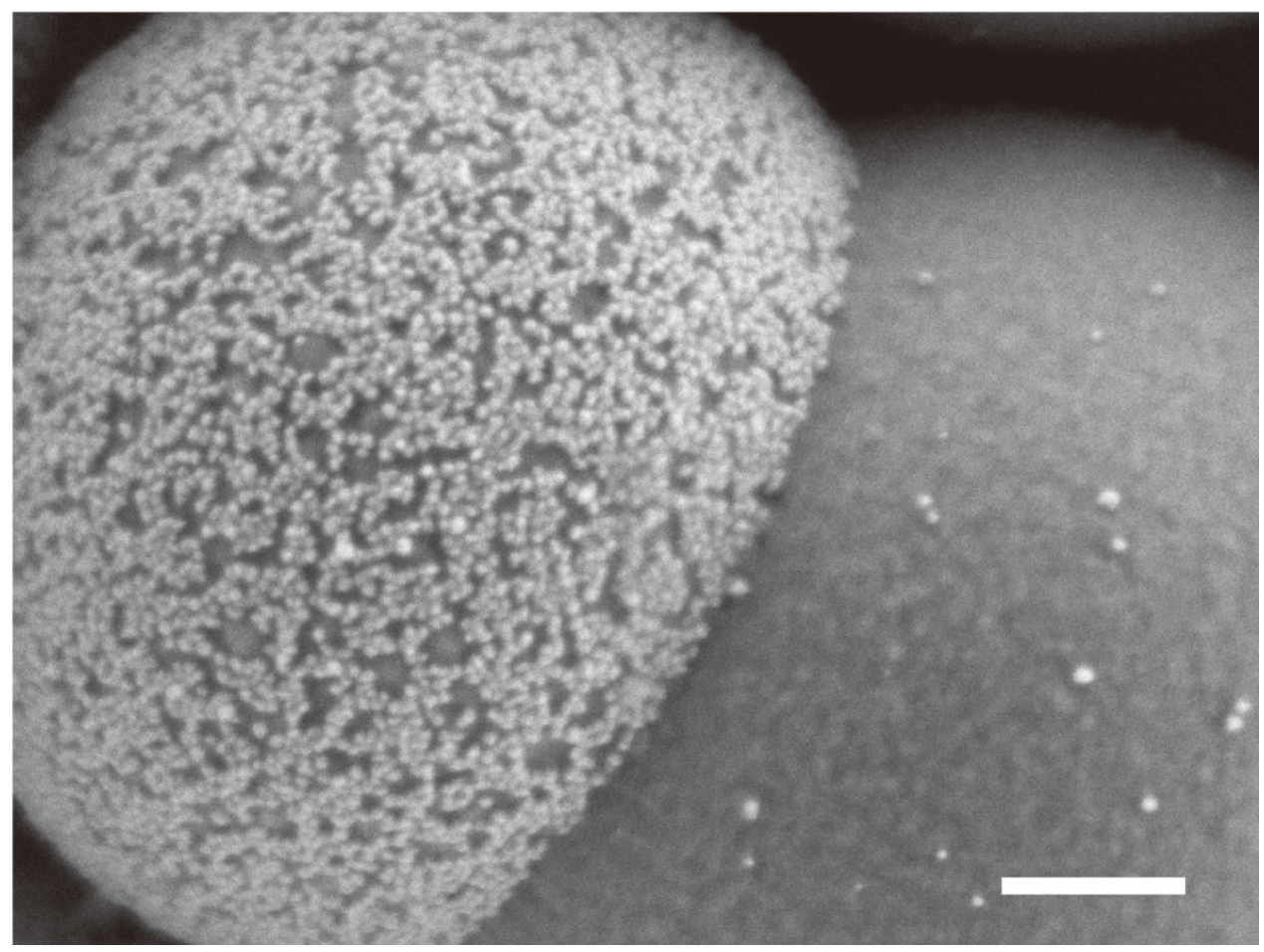

Figure S4. High-resolution SEM image of a patchy particle showing the size and morphology of gold nanoparticles plated on the particle patches. Scale bar: $200 \mathrm{~nm}$ 


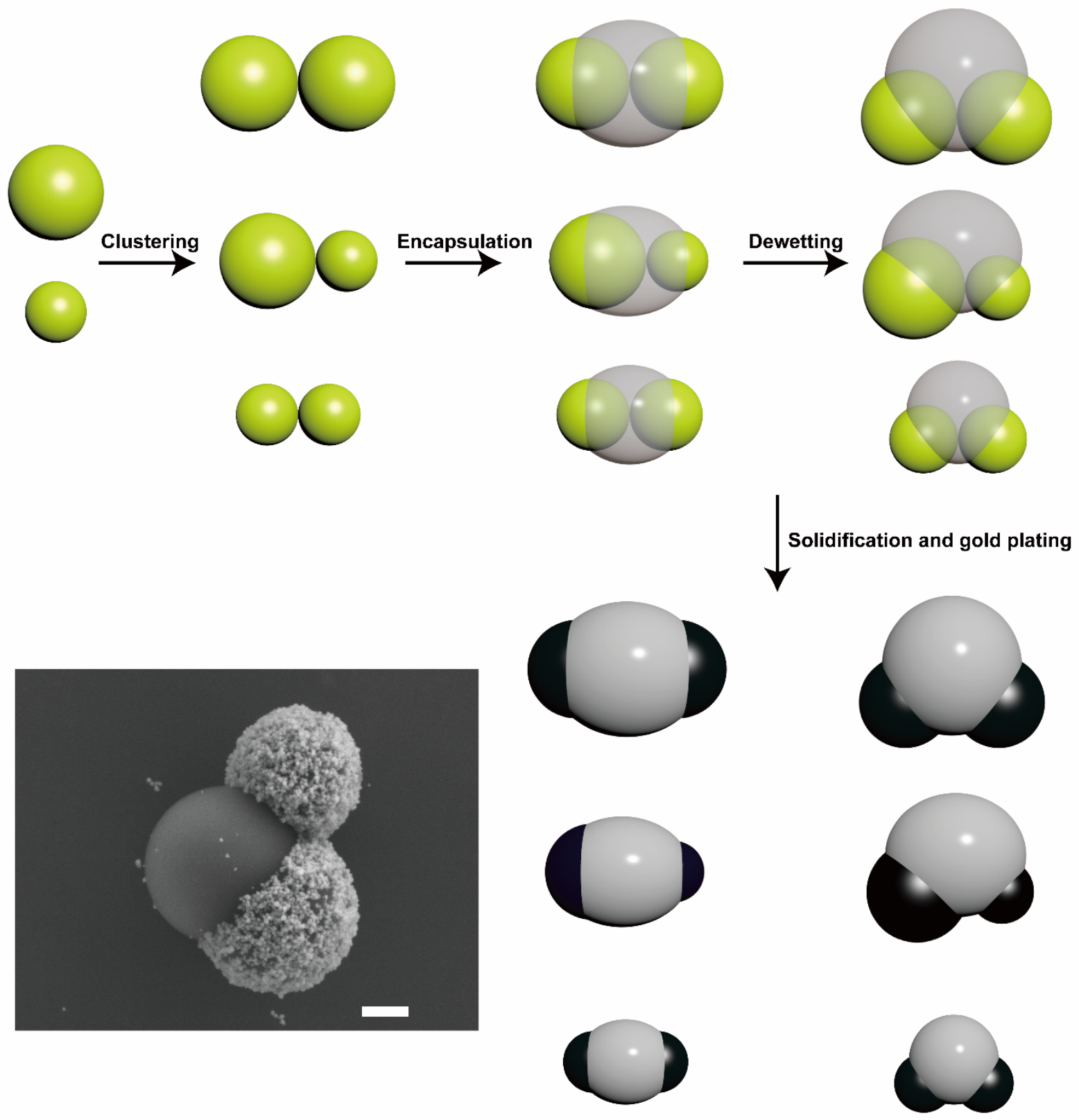

Figure S5. Schematic showing the synthesis of big-small patchy particles with both bent and linear patch configurations. SEM image shows a bent two-patch particle with a big and a small gold patch. Scale bar: $300 \mathrm{~nm}$. 

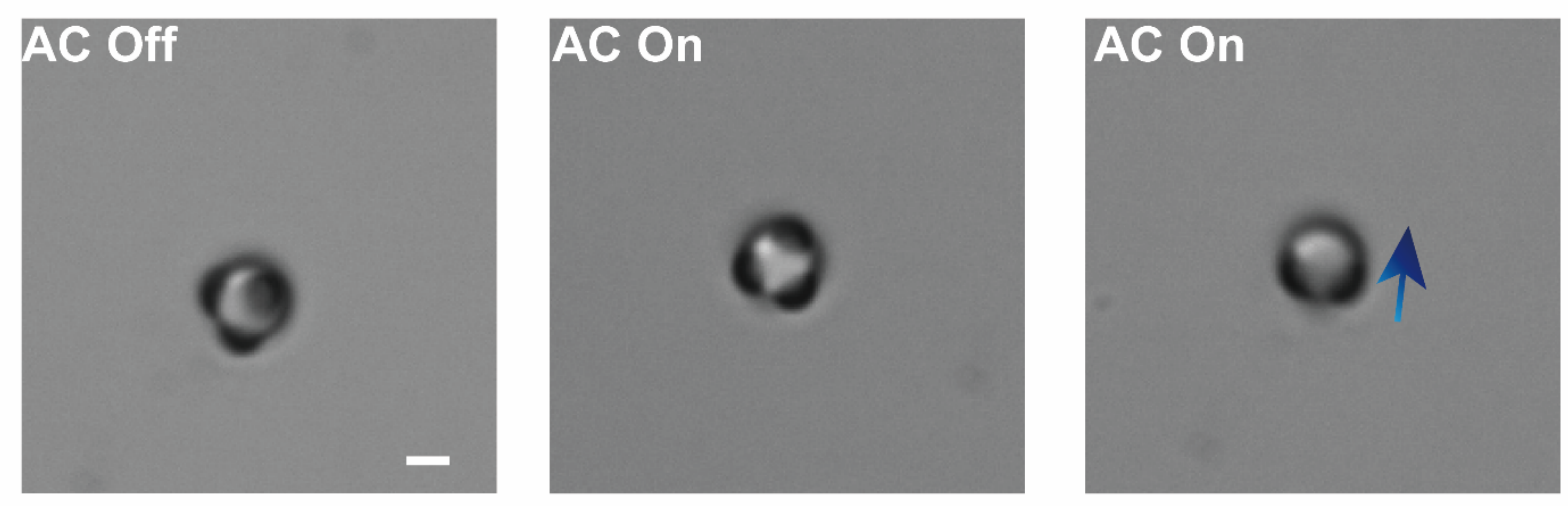

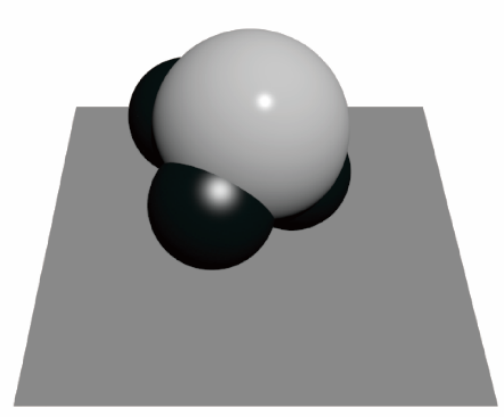

No field

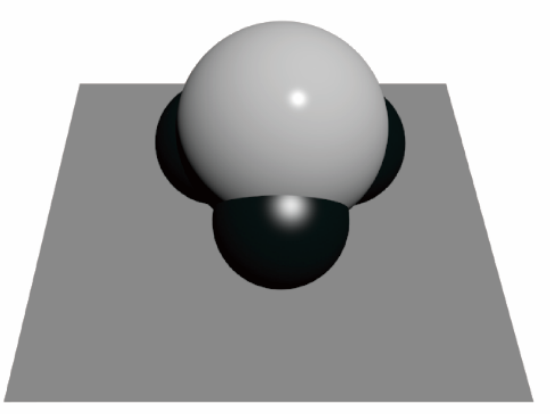

Standing

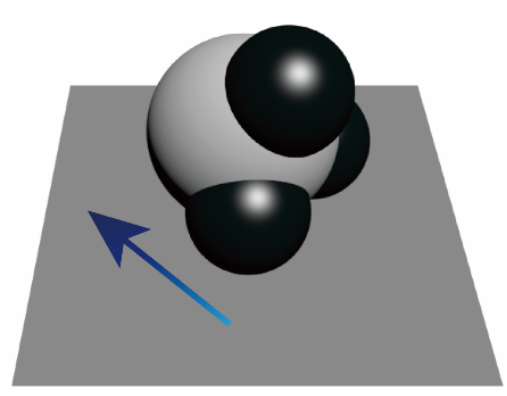

Swiming

Figure S6. Optical microscope images and cartoons showing three-patch particles when AC electric field is off (left) and on (middle and right). Depending on the initial state, the particle may "stand" or "swim" under the AC electric field. Scale bar: $1 \mu \mathrm{m}$. 


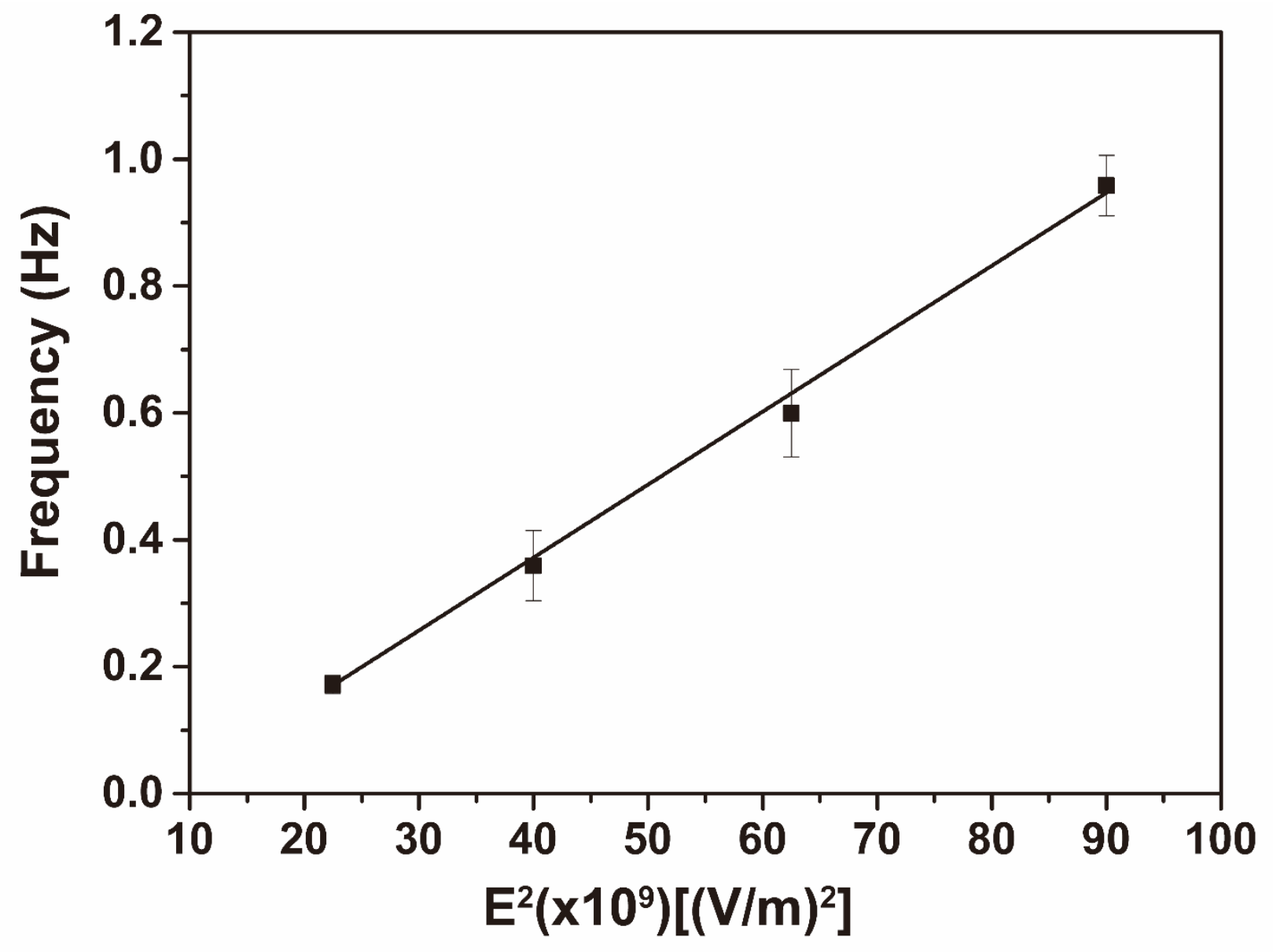

Figure S7. The frequency of $\mathbf{S}+\mathbf{P}_{2}$ steering scales linearly to the square of the strength of AC electric field. 


\section{Ligand addition}

$A B_{1} \rightarrow A B_{2}$
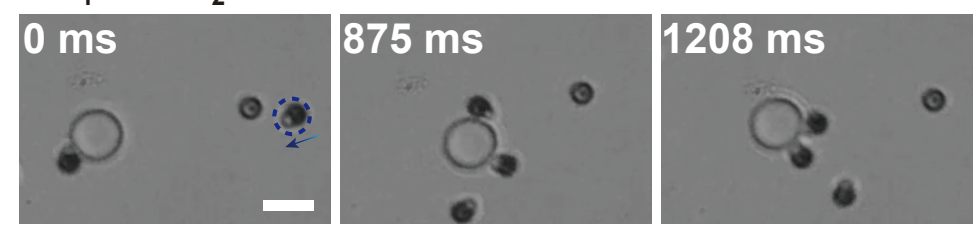

$A B_{1} \rightarrow A B_{3}$
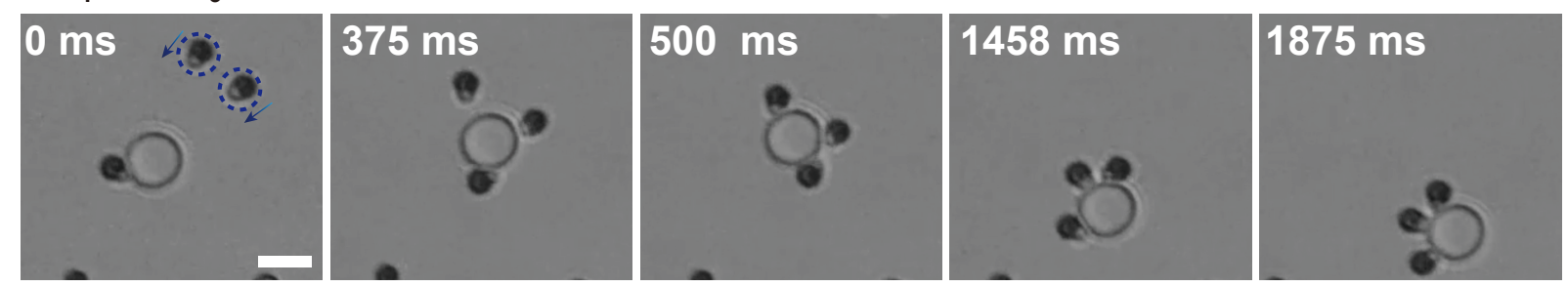

$\mathrm{AB}_{2} \rightarrow \mathrm{AB}_{3}$
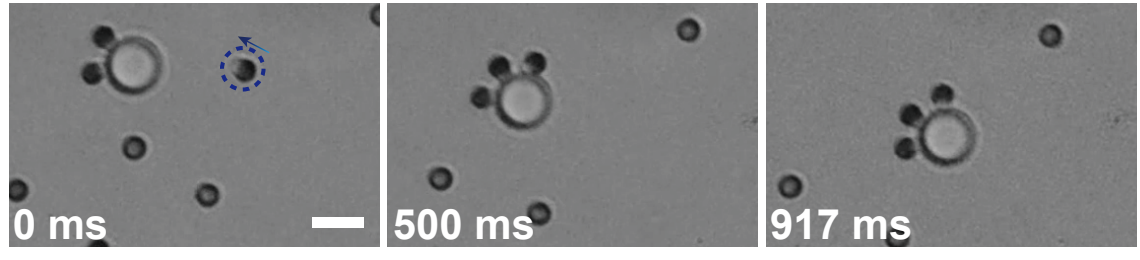

\section{Ligand deletion}

$A B_{3} \rightarrow A B_{2} \rightarrow A B_{1}$

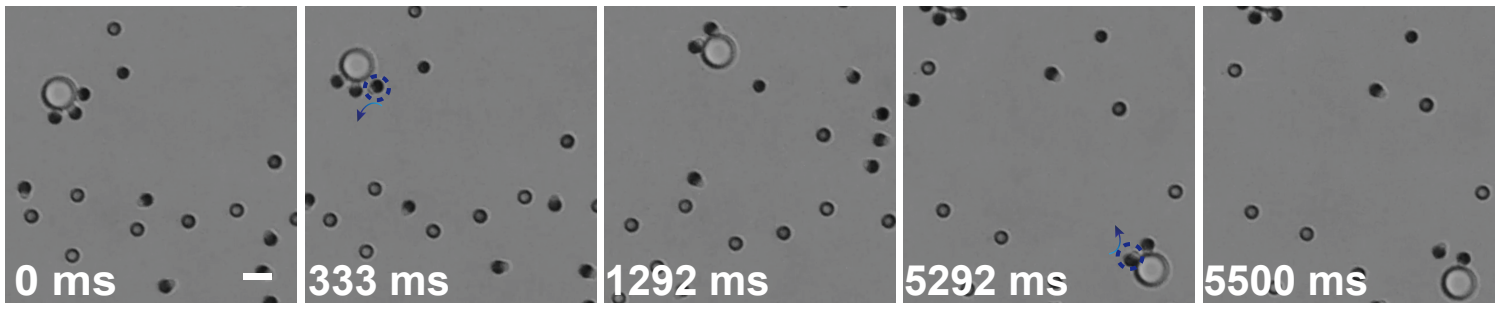

\section{Ligand exchange}

$A B_{2} \rightarrow A B_{1} \rightarrow A B_{2}$
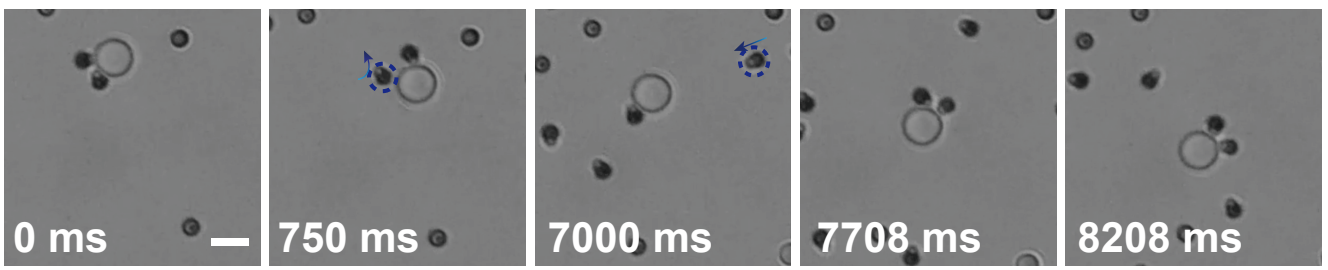

Figure S8. "Colloidal reactions"-like dynamics of $\mathbf{S}+\mathbf{P}_{2}$ assemblies. Scale bar: $4 \mu \mathrm{m}$ 


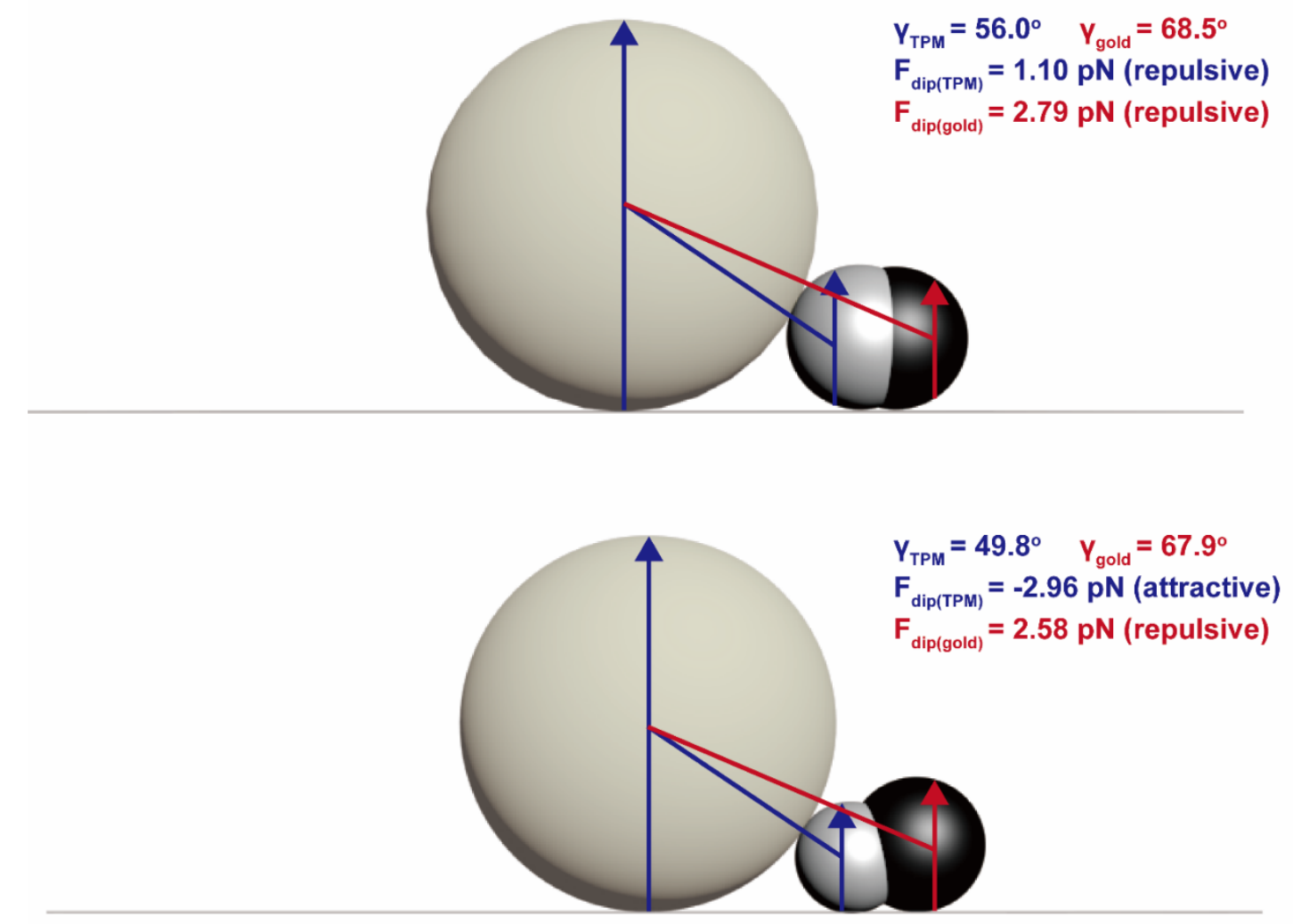

Figure S9. The dipole interactions between $\mathbf{S}$ and $\mathbf{P}_{\mathbf{1}}$ (top), $\mathbf{P}_{\mathbf{2}}$ (bottom) are schematically demonstrated and calculated according to their geometries and materials (gold/dielectrics). See more discussion on the calculation in the Supplementary Discussion section below. 


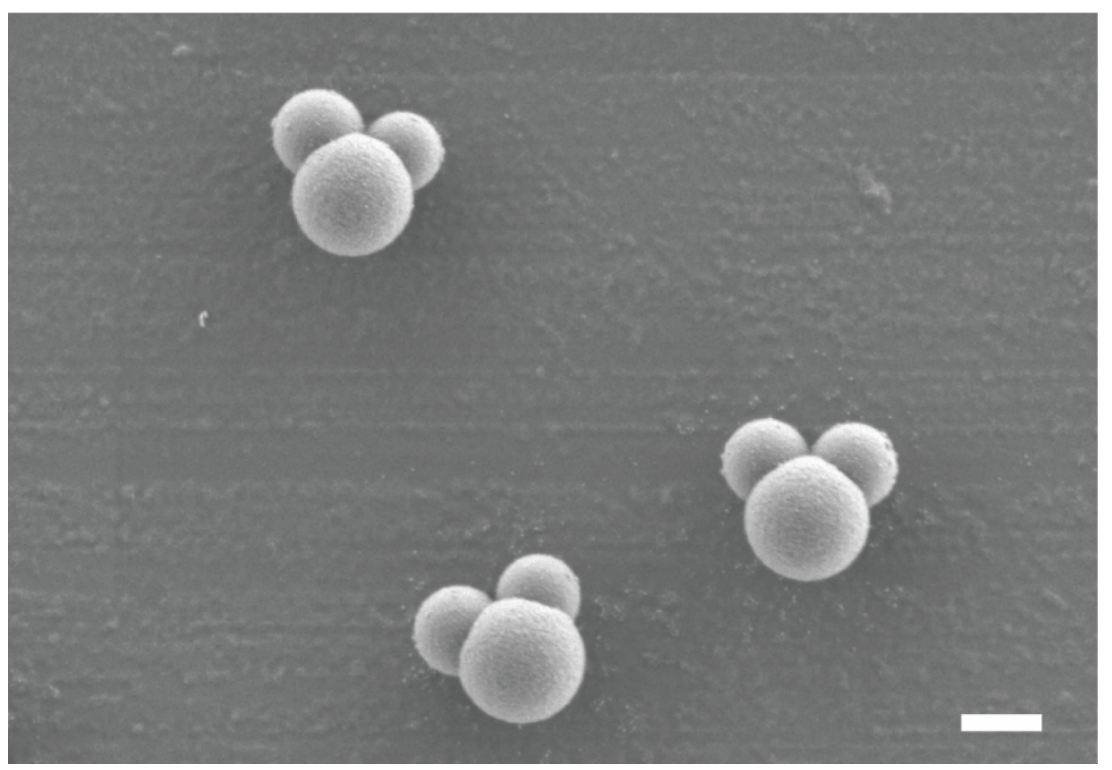

Figure S10. SEM image of purified bent two-patch particles. The purification is done by using density gradient centrifugation. Scale Bar: $1 \mu \mathrm{m}$. 


\section{SUPPLEMENTARY DISCUSSIONS}

\section{The dewetting mechanism.}

According to Young's relation:

$$
\cos \theta=\left(\gamma_{S A}-\gamma_{S O}\right) / \gamma_{O A}
$$

where $\gamma_{\mathrm{OA}}, \gamma_{\mathrm{SA}}$, and $\gamma_{\mathrm{SO}}$ are the oil-aqueous, solid-aqueous, and solid-oil interfacial energy in a wetting scenario where TPM oil droplet contacts a solid seed particle in water, as illustrated in Figure $\mathrm{S} 11$. The contact angle is $\theta$.

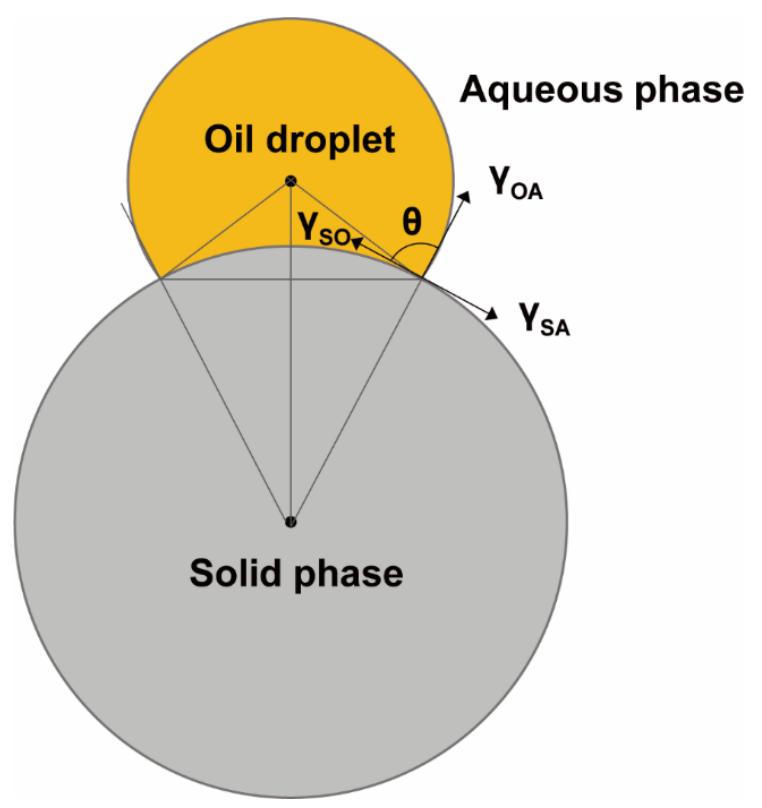

Figure S11. Illustration of the contact between oil droplet (TPM) and solid substrate (seed) in aqueous phase.

Because Triton X-100 (TX) cannot absorb to the solid-oil (SO) interface, $\gamma_{\text {so }}$ is constant. Compared to the relatively charged TPM oil droplet with a hydrophilic surface, TX prefers to adsorb onto the sparsely charged Cl-TPM substrate (i.e., the solid phase). As a result, $\gamma_{\mathrm{SA}}$ decreases dramatically while $\gamma_{\mathrm{OA}}$ only decreases slightly. The contact angle $\theta$ is therefore increased. The result can be regarded as that the addition of TX decreases the solid-aqueous surface tension, so water will spread more easily on the solid substrate, which pushes the oil to dewet the solid surface. 


\section{The steering curvature of two-patch particles.}

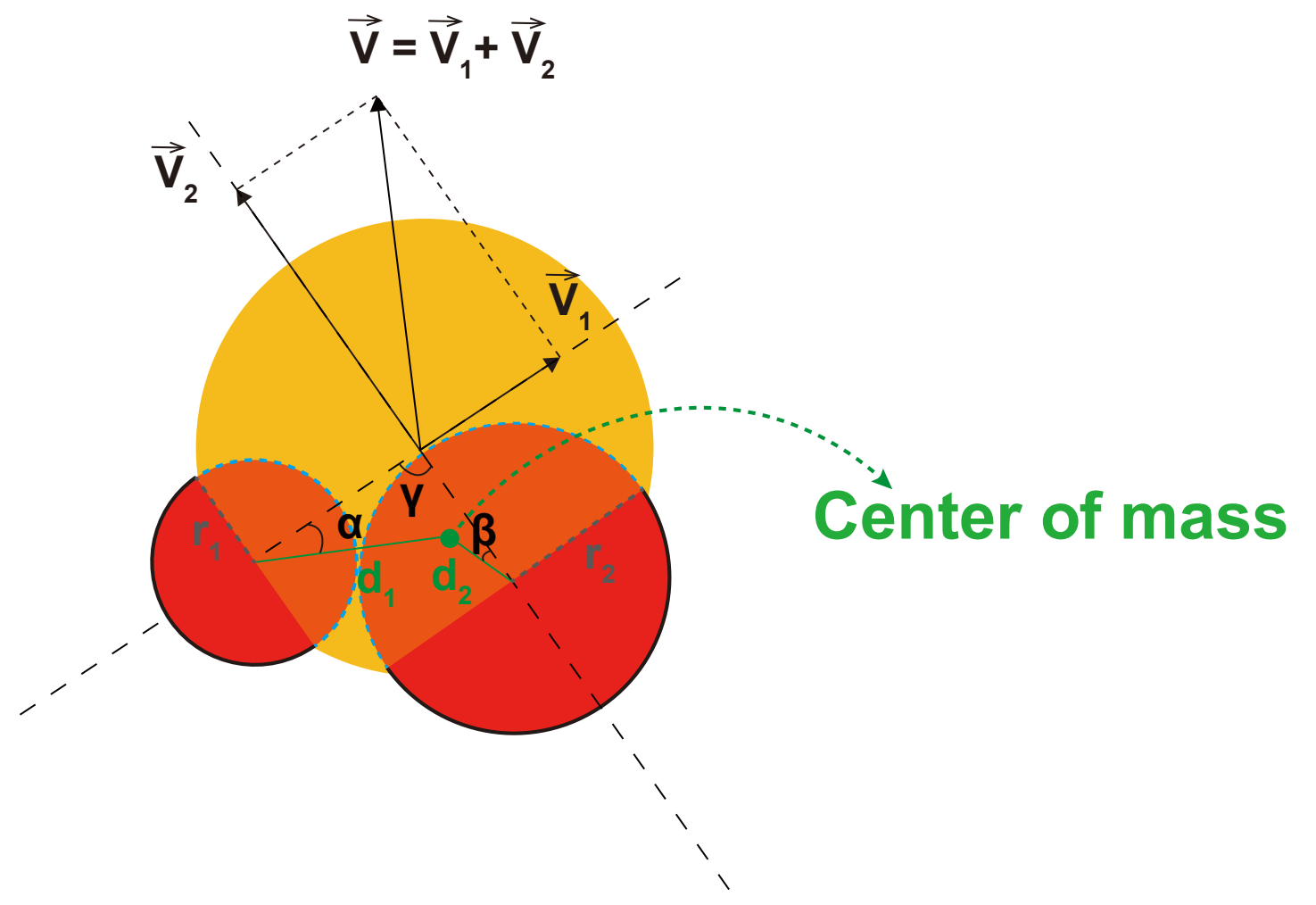

Figure S12. Illustration of a two-patch particle with a big and a small gold patch (patch shown in red).

To account for the circular motion of the two-patch particles, we analyze the steering curvature according to the particle's geometry. As shown in Figure S12, the particle can be represented with the following parts and parameters: the big sphere (yellow, radius $=R$ ), two small hemi-spheres attached to the big sphere (radius $=r_{1}, r_{2}$, and the included angle $=\gamma$ ), two gold shells covering the small hemi-spheres (thickness $=h$ ). Each part has a mass of $m_{i}$ and a position vector $\vec{a}_{i}$, pointing from the origin of coordinate to the mass center of the particle. Then, the location of the mass center for the whole patchy particle can be calculated by:

$$
\sum m_{i} * \vec{a}_{i}=m_{c} * \vec{a}_{c}(\mathrm{~S} 1)
$$

The propulsion of patchy particle is powered simultaneously by ICEP force imposed upon the two gold patches. To simplify the model, we assume that each patch drives the particle with a velocity $\vec{V}_{i}$, which is imposed on the midpoint of the patch shell. According to the ICEP mechanism $^{6}$, the velocity is proportional to the radius of the hemi-sphere that serves as patch: 


$$
\left|\vec{V}_{i}\right|=k * r_{i}
$$

where $k$ is coefficient. The angular velocity $\omega$ can be expressed as:

$$
\omega=\sin \alpha *\left|\vec{V}_{1}\right| / d_{1}-\sin \beta *\left|\vec{V}_{2}\right| / d_{2}(\mathrm{~S} 3)
$$

The overall velocity of the particle motion is:

$$
\vec{V}=\vec{V}_{1}+\vec{V}_{2}(\mathrm{~S} 4)
$$

Then, the curvature of motion can be calculated by:

$$
\rho=\frac{\omega}{|\vec{v}|}(\mathrm{S} 5)
$$

For each given $r_{1}$, the curvature of the steering can be tuned by changing the value of $r_{2}$. As shown in Figure S13, when $r_{1}=0.2 \mu \mathrm{m}$, by adjusting radius of $r_{2}$, the curvature can be tuned from $\rho=0$ (linear motion) to steering motion with curvature maximum of $0.0474 \mu \mathrm{m}^{-1}$ with the assumption that $k$ equals to $1 \mathrm{~Hz}$. When $r_{1}=0.8 \mu \mathrm{m}$, by adjusting $r_{2}$, the curvature can be tuned from $\rho=0$ (linear motion) to $-0.0467 \mu \mathrm{m}^{-1}$ (steering with reversed direction). We note that, based on experiment, $k$ value can be different.
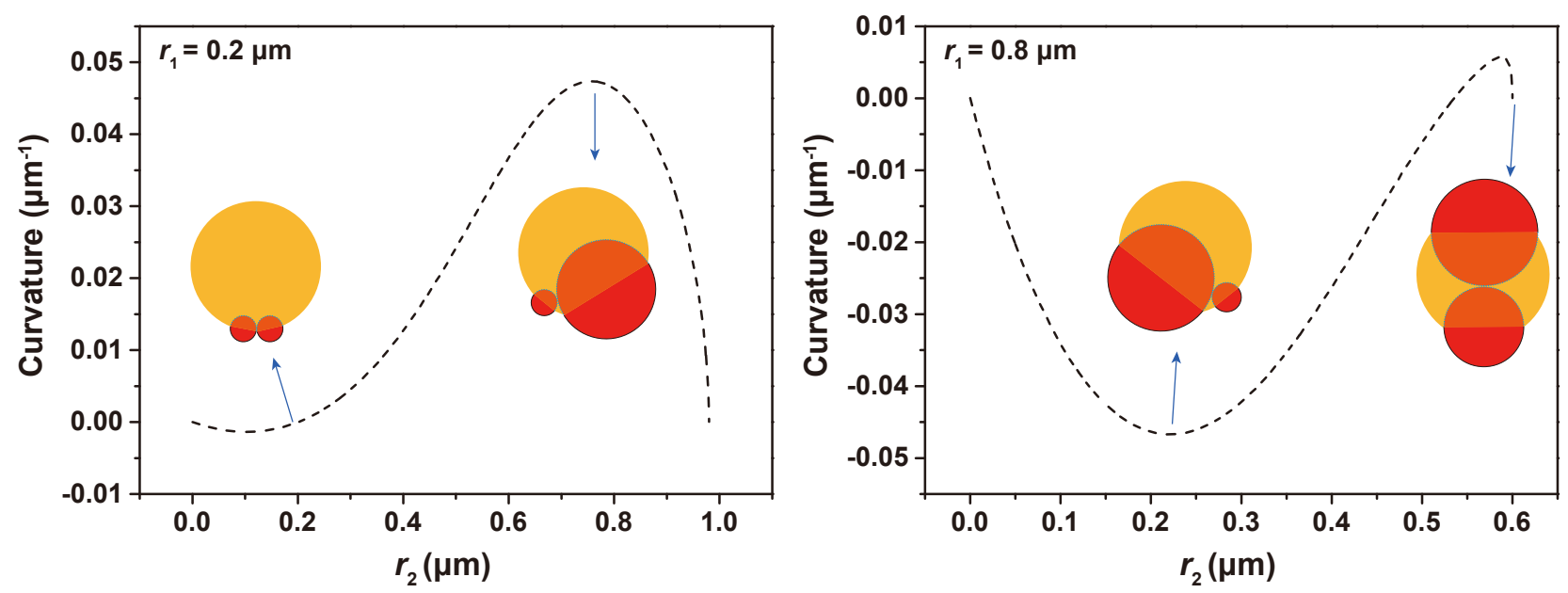

Figure S13. The calculated curvature of steering as a function of $r_{2}$ with $r_{1}$ being $0.2 \mu \mathrm{m}$ (left) and $0.8 \mu \mathrm{m}$ (right) according to equation S5 and parameters given in Table S1.

Furthermore, the extent of dewetting (or the contact angle) can be adjusted according to the concentration of TX. On one hand, this changes the size of patches and therefore changes the ICEP forces exerted on the patches (Figure 3). On the other hand, the bending angle of the two patches are changed. This provides additional handles for adjusting the curvature of steering. In 
addition, the contact angle of each of the patch on the same two-patch particle can be different, by using seed spheres with a different amount of Cl-groups, which changes the surface property. This can also be used to tune the steering behavior of two-patch particles.

Table S1. List of parameters and corresponding values.

\begin{tabular}{cc}
\hline Parameter & Value \\
\hline $\mathrm{R}$ & $1(\mu \mathrm{m})$ \\
$k$ & $1(\mathrm{~Hz})$ \\
density of polymer matrix & $1.224\left(\mathrm{~g} / \mathrm{cm}^{3}\right)$ \\
density of gold shell & $19.32\left(\mathrm{~g} / \mathrm{cm}^{3}\right)$ \\
\hline
\end{tabular}

\section{Calculation of the effective dipole shift of TPM and gold lobes}

We utilize a method proposed by Granick et $a .^{7}{ }^{7}$ to calculate the effective dipole shift of both the gold patch and the dielectric lobe. The surface polarization density $\sigma$ of a sphere (radius $R$ and dielectric constant $\varepsilon_{\mathrm{p}}$ ) under an external $\mathrm{AC}$ electric field with field strength $E_{0}$ can be described as:

$$
\sigma=3 \varepsilon_{0} E_{0}\left(\frac{\varepsilon_{p} / \varepsilon_{m}-1}{\varepsilon_{p} / \varepsilon_{m}+1}\right) \cos \theta(\mathrm{S} 6)
$$

The geometric parameters are defined as shown in Figure S14:

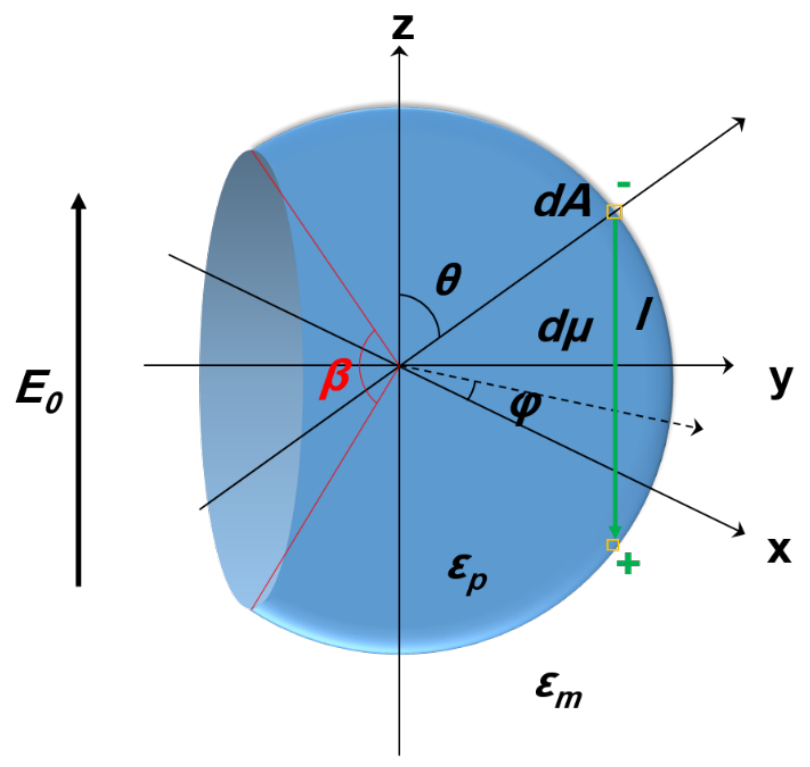

Figure S14. Dipole moment shift calculation of the spherical cap-shaped particle. 
The unit dipole moment $\mathrm{d} \mu$ of a pair of negative and positive charged surface area $\mathrm{d} A$, separated by a distance of $l$ is:

$$
\begin{gathered}
\mathrm{d} \mu(\theta, \varphi)=\sigma(\theta) \cdot d A(\theta, \varphi) \cdot l(\theta, \varphi) \\
=\left(3 \varepsilon_{0} E_{0}\left(\frac{\varepsilon_{p} / \varepsilon_{m}-1}{\varepsilon_{p} / \varepsilon_{m}+2}\right) \cos \theta\right) \cdot\left(R^{2} \sin \theta \mathrm{d} \varphi \mathrm{d} \theta\right) \cdot(2 R \cos \theta) \\
=6 \varepsilon_{0} E_{0}\left(\frac{\varepsilon_{p} / \varepsilon_{m}-1}{\varepsilon_{p} / \varepsilon_{m}+2}\right) R^{3} \cos ^{2} \theta \sin \theta \mathrm{d} \varphi \mathrm{d} \theta(\mathrm{S} 7)
\end{gathered}
$$

The effective dipole shift of the spherical cap-shaped particle can be defined as:

$$
\vec{r}_{\text {shift }}=\frac{\oint \vec{r}(\theta, \varphi) d \mu(\theta, \varphi)}{\oint d \mu(\theta, \varphi)}(\mathrm{S} 8)
$$

According to the experimental results, the $\vec{r}_{\text {shift }}$ aligns with the y axis, so the y component of displacement of the dipole $\mathrm{d} \mu$ from the center is:

$$
\delta=R \sin \theta \sin \varphi(\mathrm{S} 9)
$$

The shift of the dipole along the y axis can be calculated as:

$$
\begin{gathered}
y_{\text {shift }}=\frac{\oint \delta(\theta, \varphi) d u(\theta, \varphi)}{\oint d u(\theta, \varphi)} \\
=\frac{\left(\int_{0}^{\frac{\pi-\beta}{2}} d \theta \int_{0}^{2 \pi} d \varphi+\int_{\frac{\pi-\beta}{2}}^{\frac{\pi+\beta}{2}} d \theta \int_{0}^{2 \pi} d \varphi+\int_{\frac{\pi+\beta}{2}}^{\pi} d \theta \int_{0}^{2 \pi} d \varphi\right) \cdot 6 \varepsilon_{0} E_{0}\left(\frac{\frac{\varepsilon_{p}}{\varepsilon_{m-1}}}{\frac{\varepsilon_{p}}{\varepsilon_{m}+2}}\right) R^{4} \cos ^{2} \theta \sin ^{2} \theta \sin \varphi}{\left(\int_{0}^{\frac{\pi-\beta}{2}} d \theta \int_{0}^{2 \pi} d \varphi+\int_{\frac{\pi-\beta}{2}}^{\frac{\pi+\beta}{2}} d \theta \int_{0}^{2 \pi} d \varphi+\int_{\frac{\pi+\beta}{2}}^{\pi} d \theta \int_{0}^{2 \pi} d \varphi\right) \cdot 6 \varepsilon_{0} E_{0}\left(\frac{\frac{\varepsilon_{p}-1}{\varepsilon_{p}}}{\frac{\varepsilon_{m}}{\varepsilon_{m}+2}}\right) R^{3} \cos ^{2} \theta \sin \theta}
\end{gathered}
$$

Since the angle $\beta$ is determined by the shape of the patchy particles (obtained from SEM images), the shift of dipole along the $y$ axis is:

For mono-patch Particle 1:

$$
\begin{aligned}
& \beta_{\text {(TPM lobe) }}=148^{\circ} ; y_{\text {shift }}=0.48839 \mathrm{R} \\
& \beta_{\text {(gold lobe) }}=160^{\circ} ; y_{\text {shift }}=0.46422 \mathrm{R}
\end{aligned}
$$

For mono-patch Particle 2:

$$
\begin{aligned}
& \beta_{(\text {TPM lobe })}=140^{\circ} ; y_{\text {shift }}=0.48667 \mathrm{R} \\
& \beta_{(\text {gold lobe })}=97^{\circ} ; y_{\text {shiff }}=0.34988 \mathrm{R}
\end{aligned}
$$




\section{Calculation of induced dipole moment and interactions.}

The interactions between two complex induced dipole A and B under external AC electric field can be calculated by using equation ${ }^{8}$ :

$$
F_{\text {dip }}=\frac{3}{4} \pi \varepsilon_{0} \varepsilon_{m} a^{2} C^{2} E_{r m s}^{2}\left(\frac{2 a}{r}\right)^{4}\left[\left(3 \cos ^{2} \gamma-1\right) \hat{r}+(\sin 2 \gamma) \hat{\gamma}\right]
$$

Where $a$ is the radius of the colloidal particles, $E_{\mathrm{rms}}$ is the root mean-squared magnitude of the applied electric field, $\varepsilon_{\mathrm{m}}$ is the relative permittivity of the media, $\varepsilon_{0}$ is the vacuum permittivity, $r$ is the distance between the two dipoles, and $\gamma$ is the angle between the electric field and the connection of dipole centers.

For a spherical colloidal particle, the polarizability $C$ equals to the real part of the ClausiusMossotti (CM) function such that $C=\operatorname{Re}\{K(\omega)\} . K$ is sensitive to the frequency of external field, at the low frequency range $(2 \mathrm{kHz})$, the value of $K$ can be calculated as:

$$
\operatorname{Re}[K]=\frac{\delta_{m}-\delta_{p}}{\delta_{m}+2 \delta_{p}}(\mathrm{~S} 12)
$$

where $\delta_{\mathrm{m}}$ and $\delta_{\mathrm{p}}$ is the conductivity of media and particle. It is demonstrated that the particle conductivity results from both bulk conductivity (can be neglected for dielectric particles) and surface conductivity (derived from electro double layer). The conductivity of the gold lobe is much higher than DI water $\left(\delta_{\text {gold }}>>\delta_{\mathrm{DI}}\right) ; \operatorname{Re}[K]_{\text {gold }}$ therefore can be estimated as -0.5 . As for the dielectric lobe, the conductivity of dielectric particle is reported 6-10 times of the value of DI water ${ }^{9}$, thus we estimate the $\operatorname{Re}[K]_{\mathrm{TPM}}$ as -0.4 .

The induced dipole interactions can therefore be calculated by using equation S11. As illustrated in Figure S9, the interactions of TPM and gold coated lobes are calculated separately, taking into consideration of both their effective dipole shift and geometry induced angle $\gamma$. 


\section{VIDEO LEGEND.}

Video 1: Monopatch particles propel under $\mathrm{AC}$ electric field (frequency $f=2 \mathrm{kHz}$ ). The particles propel with their dielectric part facing forward, suggesting an induced-charge electroosmosis/electrophoresis (ICEO/ICEP) mechanism. The movie is captured and played at 24 fps.

Video 2: The propulsion of two-patch particles under AC electric field $(f=2 \mathrm{kHz})$ are shown. Particles with a linear patch configuration barely move due to the balanced ICEP forces. Particles with a bent patch configuration propel with a speed depending on the bent angle and patch size. The movie is captured and played at $24 \mathrm{fps}$.

Video 3: Asymmetrical two-patch particle with a big and a small patch steers under AC electric field $(f=2 \mathrm{kHz})$. The steering direction (clockwise or counterclockwise) may change as the particle flips when the field is off. The movie is captured and played at $24 \mathrm{fps}$.

Video 4: The assembly of $\mathbf{S}+\mathbf{P}_{\mathbf{1}}$ under AC electric field $(f=10 \mathrm{kHz})$ is shown, which ultimately form $\mathbf{S}+2 \mathbf{P}_{1}$ assembly which spins with both clockwise and counterclockwise directions. The movie is captured and played at $24 \mathrm{fps}$.

Video 5: Under AC electric field $(f=10 \mathrm{kHz}), \mathbf{P}_{\mathbf{2}}$ "stand up" and align its long axis to the direction of electric field resulting in in-plane repulsion with $\mathbf{S}$ that prevents any assembly. The movie is captured and played at $24 \mathrm{fps}$.

Video 6: Under AC electric field $(f=2 \mathrm{kHz}), \mathbf{P}_{\mathbf{1}}$ approaches and touches $\mathbf{S}$, but would immediately escape from $\mathbf{S}$, leading to no assembled structures. The movie is captured and played at 24 fps.

Video 7: Under AC electric field $(f=2 \mathrm{kHz}), \mathbf{P}_{\mathbf{2}}$ can couple with $\mathbf{S}$ when they encounter. Once assembled, they together steer with both directions. A larger view containing multiple such 
steering assemblies are also shown in the later part of the movie. The movie is captured and played at $24 \mathrm{fps}$.

Video 8: Movie showing the "colloidal reaction"-like dynamics of assemblies based on $\mathbf{P}_{\mathbf{2}}$ and $\mathbf{S}$ under $\mathrm{AC}$ electric field $(f=2 \mathrm{kHz})$. Various pathways and reconfiguring structures are shown. The movie is captured and played at $24 \mathrm{fps}$.

Video 9: Movie showing the dynamics of $\mathbf{P}_{2}$ in the presence of $\mathbf{S}$ under AC electric field $(f=2 \mathrm{kHz})$. $\mathbf{P}_{2}$ patchy particle can escape from one assembly and re-join another assembly. The movie is captured and played at $24 \mathrm{fps}$.

Video 10: When two-patch particles with a big and small gold patch assembles with dielectric sphere $\mathbf{S}$, they bind to the sphere only with their small patch (under AC electric field, $f=1 \mathrm{kHz}$ ). The assembly steers after the binding. The movie is captured and played at $24 \mathrm{fps}$. 


\section{REFERENCES}

1. Wang, Y.; Wang, Y.; Zheng, X.; Ducrot, E.; Yodh, J. S.; Weck, M.; Pine, D. J., Crystallization of DNA-coated colloids. Nat Commun 2015, 6, 7253.

2. Yi, G. R.; Manoharan, V. N.; Michel, E.; Elsesser, M. T.; Yang, S. M.; Pine, D. J., Colloidal Clusters of Silica or Polymer Microspheres. Advanced Materials 2004, 16 (14), 12041208.

3. Manoharan, V. N.; Elsesser, M. T.; Pine, D. J., Dense packing and symmetry in small clusters of microspheres. Science 2003, 301 (5632), 483-7.

4. Lin, C.-H.; Chen, Y.-L.; Jiang, H.-R., Orientation-dependent induced-charge electrophoresis of magnetic metal-coated Janus particles with different coating thicknesses. RSC $A d v$. 2017, 7 (73), 46118-46123.

5. Brakke, K. A., The Surface Evolver. Experimental Mathematics 1992, 1 (2), 141-165.

6. Gangwal, S.; Cayre, O. J.; Bazant, M. Z.; Velev, O. D., Induced-charge electrophoresis of metallodielectric particles. Phys Rev Lett 2008, 100 (5), 058302.

7. Zhang, J.; Yan, J.; Granick, S., Directed Self-Assembly Pathways of Active Colloidal Clusters. Angew Chem Int Ed Engl 2016, 55 (17), 5166-9.

8. Mittal, M.; Lele, P. P.; Kaler, E. W.; Furst, E. M., Polarization and interactions of colloidal particles in ac electric fields. J Chem Phys 2008, 129 (6), 064513.

9. Shields, C. W.; Han, K.; Ma, F.; Miloh, T.; Yossifon, G.; Velev, O. D., Supercolloidal Spinners: Complex Active Particles for Electrically Powered and Switchable Rotation. Advanced Functional Materials 2018, 28 (35), 1803465. 